\title{
Exosome secreted from adipose-derived stem cells attenuates diabetic nephropathy by promoting autophagy flux and inhibiting apoptosis in podocyte
}

\author{
Juan Jin ${ }^{1,2,3 \dagger}$, Yifen Shi ${ }^{1,4 \dagger}$, Jianguang Gong ${ }^{2,3}$, Li Zhao ${ }^{2,3}$, Yiwen Li ${ }^{2,3}$, Qiang He ${ }^{2,3^{*}}$ (D) and He Huang ${ }^{1 *}$
}

\begin{abstract}
Background: It is confirmed that adipose-derived stem cells (ADSCs) transplantation effectively relieves kidney fibrosis and type 2 diabetes disease in mice. Currently, exosome from urine-derived stem cells (USCs) can protect type 1 diabetes-mediated kidney injury and attenuate podocyte damage in diabetic nephropathy (DN). Exosome derived from USCs has evolved into the strategy for DN treatment, but the role of ADSCs-derived exosome (ADSCsExo) in DN remains unclear. The present study is aimed to investigate the therapeutic action and molecular mechanism of ADSCs-derived exosome on DN.

Methods: ADSCs and exosome were authenticated by immunofluorescence and flow cytometry. Morphology and the number of exosome were evaluated by electron microscope and Nanosight Tracking Analysis (NTA), respectively. Cell apoptosis was assessed using flow cytometry. Podocyte autophagy and signaling transduction were measured by immunofluorescence and immunoblotting. Dual Luciferase Reporter assay was employed to detect the regulatory relationship between miR-486 and Smad1.

Results: ADSCs-Exo attenuated spontaneous diabetes by reducing levels of urine protein, serum creatinine (Scr), blood urea nitrogen (BUN), and podocyte apoptosis in mice. In in vitro experiment, ADSCs-Exo also reversed high glucoseinduced decrease of cell viability and the increase of cell apoptosis in MPC5 cells. In terms of mechanism, ADSCs-Exo could enhance autophagy flux and reduce podocyte injury by inhibiting the activation of mTOR signaling in MPC5 and spontaneous diabetic mice. Eventually, we found that miR-486 was the key factors in ADSCs and in the process of ADSCs-Exo-mediated improvement of DN symptom in vivo and in vitro. miR-486 reduced Smad1 expression by target regulating Smad1 whose reduction could inhibit mTOR activation, leading to the increase of autophagy and the reduction of podocyte apoptosis.

Conclusions: In conclusion, we illustrated that ADSCs-Exo vividly ameliorated DN symptom by enhancing the expression of miR-486 which led to the inhibition of Smad1/mTOR signaling pathway in podocyte. Possibly, ADSCs-Exo was used as a main therapeutic strategy for DN in future.
\end{abstract}

Keywords: Adipose-derived stem cells, Exosome, Podocyte damage, Cell autophagy, miR-486, Smad1/mTOR signaling

\footnotetext{
*Correspondence: qianghe1973@126.com; huanghe@zju.edu.cn

†Juan Jin and Yifen Shi contributed equally to this work.

2Department of Nephrology, Zhejiang Provincial People's Hospital,

Hangzhou, Zhejiang 310014, People's Republic of China

${ }^{1}$ Bone Marrow Transplantation Center, The First Affiliated Hospital, Zhejiang

University School of Medicine, Hangzhou, Zhejiang 310003, People's Republic

of China

Full list of author information is available at the end of the article
}

(c) The Author(s). 2019 Open Access This article is distributed under the terms of the Creative Commons Attribution 4.0 International License (http://creativecommons.org/licenses/by/4.0/), which permits unrestricted use, distribution, and reproduction in any medium, provided you give appropriate credit to the original author(s) and the source, provide a link to the Creative Commons license, and indicate if changes were made. The Creative Commons Public Domain Dedication waiver (http://creativecommons.org/publicdomain/zero/1.0/) applies to the data made available in this article, unless otherwise stated. 


\section{Background}

Diabetic nephropathy (DN) is a complex and common chronic kidney disease worldwide [1]. The clinical features of DN are characterized by elevation of persistent albuminuria, thickness of glomerular basement membrane (GBM), and aggregation of the extracellular matrix, which results in autophagy flux inhibition, podocyte damage promotion, and progressive renal dysfunction [2]. However, the pathogenesis of podocyte injury-mediated DN remains vague and therapeutic strategies for DN are lacking.

Podocyte damage is a key regulator and can be used as a clinical predictor in the evolution of $\mathrm{DN}[3,4]$. Homeostasis maintenance under pathophysiological stress is extremely important in determining the fate of podocytes [5]. Podocyte autophagy is mainly responsible for protein and organelle degradation following by regulating cell homeostasis, whose dysfunction is regarded as an essential induction factor of podocyte injury $[6,7]$. Numerous signaling molecules are involved in the process of podocyte autophagy including mTOR (mechanistic target of rapamycin) and AMP-activated protein kinase (AMPK) $[8,9]$. In fact, mTOR signaling activation is closely linked to the accelerated podocyte injury in DN patients which potentially suggests an involvement of mTOR signaling-mediated autophagy dysfunction in DN disease $[10,11]$. Collectively, autophagy flux might be a therapeutic target in podocyte damage-derived DN disease.

Previous studies have demonstrated that mesenchymal stem cells (MSCs) are a therapeutic candidate for halting the progression of DN [12]. There have been identified that adipose-derived stem cells (ADSCs) transplantation can alleviate acute kidney injury by enhancing cell repair and regeneration abilities [13]. ADSCs transplantation also attenuates podocyte damage in $\mathrm{DN}$ by activating klotho and inhibiting Wnt/ $\beta$-catenin pathway [14]. Though many researchers have confirmed that ADSCs possesses the repair effect on cell/tissue injury, these protective effect is depended on the paracrine of ADSCs due to the low rate of effector cells differentiated from ADSCs after transplantation. Paracrine cytokines and exosome derived from ADSCs act as the key repair factors for wound healing $[15,16]$. Especially, ADSCs-derived exosome (ADSCsExo) are internalized into fibroblasts to improve cutaneous wound healing by promoting cell migration, proliferation, and collagen synthesis [17]. Besides, exosome secreted by urine-derived stem cells have the potential to promote renal repair by repressing podocyte apoptosis in diabetic rats [18]. These studies suggest that exosome secreted from stem cells is the key paracrine regulator for cell/tissue repair. However, whether ADSCs-Exo possesses the protective effect on podocyte injury in DN and acts as a bridge between autophagy and apoptosis in podocyte damage are still not clearly understood.
Herein, ADSCs and ADSCs-Exo were isolated successfully from $\mathrm{C} 57 \mathrm{BL} / \mathrm{Ks} \mathrm{db} / \mathrm{m}$ mice and ADSCs-Exo administration reduced cell apoptosis through regulating mTOR signaling-mediated autophagy flux in DN mice and high glucose-induced MPC5 cells. Mechanismly, miR-486 of ADSCs-Exo acted the key improvement role on renal injury through weakening mTOR activation-mediated autophagy via directly targeting smad1. Possibly, ADSCsExo might be served as a main treatment strategy for DN in future.

\section{Materials and methods Materials}

Mannitol (SM8120) was obtained from Solarbio. RPMI-1640 (SH30809.01B), DMEM/F12 medium (SH30023.01B), and BSA (SH30574.03) were purchased from Hyclone. Collagenases I (17100-017), II (17101-015), IV (17104-019), and 0.25\% Trypsin (15050065) were purchased from Gibco. PI (25535-16-4), RNase A (9001-99-4), dexamethasone (D4902), $\beta$-glycerophosphoric acid (G9422), ascorbic acid (A7562), insulin (I5500), indomethacin (I7378), and 3 isobutyl 1 methylxanthine (IBMX; I7018) were from Sigma. 1\% Triton $\mathrm{x}-100$ was obtained from SBJBio Life Sciences (SBJ-1141).

\section{Animal treatment}

Eight-week-old C57BL/KsJ db/m (control mice, $n=10$ ) and $\mathrm{C} 57 \mathrm{BL} / \mathrm{KsJ} \mathrm{db} / \mathrm{db}$ (spontaneous diabetes mice, $n=20$ ) male mice were obtained from Cavens Lab Animal (SCX, 2016-0010, Changzhou, China). All animal care procedures were approved by Laboratory Animals of Zhejiang Provincial People's Hospital. The mice were housed three to five per cage in a room under controlled light $(12 \mathrm{~h} /$ day) and temperature $\left(22 \pm 2{ }^{\circ} \mathrm{C}\right)$ conditions with free access to food and water. At 12 weeks old, periodic acid-Schiff (PAS) staining was performed to for histopathology investigation to verify disease phenotype of DN according to previous study. At 13 weeks old, mice were treated with tail intravenous injection of PBS or ADSCs-Exo for additional 12 weeks.

\section{Cell isolation, culture, and treatment}

Eight-week-old C57BL/KsJ db/m male mice was anesthetized using pentobarbital (200 mg/kg i.p.), and subcutaneous adipose tissue in groin was harvested. ADSCs were isolated by utilizing collagenase digestion method. Briefly, adipose tissue was minced and washed with PBS buffer twice followed by centrifugation at $1200 \mathrm{rpm}$ for $10 \mathrm{~min}$. The supernatant was removed, and the mixed collagenases were added into the precipitate. After digestion for $40 \mathrm{~min}$ at $37^{\circ} \mathrm{C}$, complete medium was added to stop the reaction and the digested mixture was filtered through 40-mm cell strainer and centrifuged at 1500 $\mathrm{rpm}$ for $8 \mathrm{~min}$. The precipitate was then resuspended in 
RPMI- 1640 complete medium and cultured at $37^{\circ} \mathrm{C}$ in a humidified incubator in 5\% $\mathrm{CO}_{2}$. In miRNA-486 inhibitor assay, ADSC cells were transfected with miRNA-486 inhibitor and negative control in ADSCs for $48 \mathrm{~h}$. Then, ADSCs-Exo were isolated for the next experiments. The sequences of miRNA-486 inhibitor and negative control were as follows: AUCCUGUACUGAGCUGCCCCG and CAG UAC UUU UGU GUA GUA CAA.

Mouse podocyte MPC5 cells were purchased from Institute of Basic Medical Sciences, Chinese Academy of Medical Sciences (3111C0001CCC000230). MPC5 cells were cultured in DMEM medium containing with $10 \%$ fetal bovine serum and incubated at $37^{\circ} \mathrm{C}$ in a humidified atmosphere containing 5\% CO2. For CCK8 assay, MPC5 cells were treated with $5.5 \mathrm{mM}$ D-glucose (NG), $5.5 \mathrm{mM}$ D-glucose+ $24.5 \mathrm{mM}$ mannitol (MA), $30 \mathrm{mM}$ D-glucose (HG), and combination of HG and concentration gradient ADSCs-Exo for $24 \mathrm{~h}, 48 \mathrm{~h}, 72 \mathrm{~h}$, and $96 \mathrm{~h}$. For apoptosis and autophagy experiments, MPC5 cells were treated with NG, MA, HG, and combination of $\mathrm{HG}$ and ADSCs-Exo for $48 \mathrm{~h}$.

\section{Osteogenic and adipogenic differentiation and authentication of ADSCs}

ADSCs in passage 3 were subjected to stem cell induction and appraisal system for examining differentiation ability of ADSCs. For the ability of osteogenic differentiation, $1 \times 10^{5}$ cells were seeded on coverslip in six-well plate. After incubation for $24 \mathrm{~h}$, cells were induced with osteogenic medium containing 10\% FBS, $10 \mathrm{nM}$ dexamethasone, $10 \mathrm{mM} \beta$-glycerophosphate, and $50 \mu \mathrm{g} / \mathrm{mL}$ ascorbic acid in high-glucose DMEM medium. Normal high-glucose DMEM was served as the control group. At day 14, the induced cells were harvested for adipogenic assessments by utilizing alkaline phosphatase (ALP) detection kit (A059-2, Jiangcheng biotechnology, Nanjing, China) and Alizarin Red Staining. The ability of ALP was according to the manufacturer's instruction. ALP activity was measured at the wavelength of $520 \mathrm{~nm}$, and activity relative to control was calculated after normalization to the total protein content. Alizarin Red Staining was another employed method for detecting mineralization after osteogenic differentiation. In brief, the induced ADSCs on the glass were washed with PBS for three times and fixed with $4 \%$ paraformaldehyde for $15 \mathrm{~min}$. Next, the glass was stained with $0.2 \%$ Alizarin Red for $20 \mathrm{~min}$. After washing with PBS, staining results were observed and taken photographs under optical microscope. The positive cells presented as nacarat.

For adipogenic differentiation of ADSCs, cells in passage 3 were incubated with adipogenic medium containing $10 \% \mathrm{FBS}, 1 \mu \mathrm{M}$ dexamethasone, $60 \mu \mathrm{M}$ indomethacin, $500 \mathrm{nM}$ IBMX, and $10 \mu \mathrm{g} / \mathrm{m}$ insulin in high-glucose DMEM medium. Oil Red $O$ staining was used for quantitatively adipogenic assessments as per previous study. Briefly, cells were washed with PBS for twice and fixed using $4 \%$ paraformaldehyde for $5 \mathrm{~min}$. Then, cells were washed again twice and stained in propylene glycol for $5 \mathrm{~min}$, Oil Red $\mathrm{O}$ solution for $15 \mathrm{~min}$ at $60^{\circ} \mathrm{C}$. After Oil Red O solution was removed, cells were differentiated using $85 \%$ propylene glycol for $5 \mathrm{~min}$. Next, cells were stained with hematoxylin for 30s. After washing again, results were observed and taken with optical microscopes.

\section{Isolation of exosome}

Passage 3 ADSCs were seeded into 6- $\mathrm{cm}$ dish and incubated in a $37^{\circ} \mathrm{C}$ incubator containing $5 \% \mathrm{CO} 2$. When grown to $70-80 \%$ confluence, cells were cultured with fresh medium containing with exosome-free FBS for another $24 \mathrm{~h}$ which was obtained by ultracentrifugation at $100,000 \mathrm{~g}$ for $18 \mathrm{~h}$ followed by filtration through a $0.22-\mu \mathrm{m}$ filter. After $24 \mathrm{~h}$ of incubation, the conditioned medium (CM) was collected and exosome was isolated by utilizing exosome extraction kit (Wako pure Chemicals Industry, 293-77601) according to the manufacturer's protocol. Briefly, $30 \mathrm{ml} \mathrm{CM}$ were centrifuged at $300 \times g$ for $5 \mathrm{~min}, 1200 \times g$ for $20 \mathrm{~min}$, and then $10,000 \times g$ for $30 \mathrm{~min}$ at $4{ }^{\circ} \mathrm{C}$ to remove cells, debris, and large extracellular vesicles (EVs). Next, the CM were concentrated to $1 \mathrm{~mL}$ by using Amicon Ultra-15 Ultracel-100 K (Merck KGaA, Darmstadt, Germany, UFC910024). Then concentrated $\mathrm{CM}$ were incubated with Streptavidin Magnetic Beads $(60 \mathrm{mg})$ accompanied by additional $1 \mu \mathrm{g}$ of biotinylated mouse Tim4-Fc, $350 \mu \mathrm{L}$ Exosome Capture Immobilizing Buffer, and $50 \mu \mathrm{L}$ Exosome Binding Enhancer overnight at $4{ }^{\circ} \mathrm{C}$. The next day, the beads were washed with washing buffer for three times and the bound EVs were eluted by utilizing Exosome Elution Buffer.

\section{Transmission electron microscopy (TEM)}

Exosome morphology was analyzed using TEM analysis kit (E1610, Weihui Biotechnology, Peking, China). Firstly, exosome was placed on parafilm membrane as a $10 \mu \mathrm{L}$ drop of exosome suspension and the EM membrane covered with formvar carbon was put up on the suspension for $10 \mathrm{~min}$ to adsorb exosome as much as possible. After washing with wash buffer, EM containing exosome was observed by transmission electron microscopy (JEOL, JSM-IT300LV) and images were taken using an electron sensitive Olympus KeenView CCD camera.

\section{Nanoparticle tracking analysis (NTA)}

Exosome was diluted to a volume of $1 \mathrm{~mL}$ in TPM to analysis. Size and concentration of exosome were determined through Nanosight Tracking Analysis by utilizing ZetaView PMX 110 (Particle Metrix, Meerbusch, Germany) according to previous protocol [19]. 


\section{Urine protein, serum creatinine (Scr), and blood urea nitrogen (BUN) measurement}

Urine was collected after 12 weeks of exosome treatment and measured using BCA kit. In brief, mixture of $\mathrm{BCA}$ working solution and urine was co-incubated at 37 ${ }^{\circ} \mathrm{C}$ for $30 \mathrm{~min}$. The OD value was tested at the wavelength of $562 \mathrm{~nm}$. In the end of exosome treatment, blood plasma was collected by abdominal aortic method and stand for $2 \mathrm{~h}$ at room temperature. After centrifugation at $3500 \mathrm{rpm}$ for $5 \mathrm{~min}$, Scr and BUN levels were measured through detection kits (Scr: C011-1, Jiangcheng Bio, Nanjing; BUN: C013-2, Jiangcheng, Nanjing), respectively.

\section{Plasmid construction and cell transfection}

For luciferase reporter plasmids, Smad1-3'UTR-wt (wild type) and Smad1-3'UTR-mut (mutant) fragments were inserted into pYr-MirTarget vector. For Smad1-overexpressed plasmid construction, mus Smad1 open reading frame fragment was inserted into pcDNA3.1 vector. Next, recombinant plasmids were transferred into $\mathrm{DH} 5 \alpha$ coli cells and screened by clone assay. PCR amplification primers were as follows: Smad1-3'UTR-wt, forward: GGTTCTTTTCCAACGCTATT, reverse: CACTTCAGA AAGACTATCAG; Smad1-3'UTR-mut, forward: TTTG TTTGTTTTTAATGAAGACGTTAATCGTTATGACAT GCATAG, reverse: ATAACGATTAACGTCTTCA TTAAAAACAAACAAAAAACCCATTCA. After sequencing, the reporter plasmids were extracted by utilizing plasmid extraction kit.

\section{Dual luciferase reporter assay}

HEK-293 T cells were seeded on 24-well plate and incubated for $24 \mathrm{~h}$. Then, cells were transfected with Smad1-3'UTR reporter and miR-486 mimic NC/ miR-486 mimic plasmids for another $48 \mathrm{~h}$. Culture medium was removed, and cells were washed with PBS for twice. Cells were lysed through adding PLA buffer, and luciferase activity was measured by utilizing dual luciferase reporter assay system (E1910, Promega). OD value was observed through microplate reader.

\section{Immunofluorescence (IF)}

Cells were fixed in $4 \%$ paraformaldehyde for $30 \mathrm{~min}$ followed by washing for three times using PBS. Cells were treated with $0.1 \%$ Triton $\mathrm{X}-100$ for $15 \mathrm{~min}$ and blocked using 5\% BSA (Hyclone, SH30574.03) for $1 \mathrm{~h}$. For renal tissue, specimen was fixed in $4 \%$ paraformaldehyde for $24 \mathrm{~h}$ or more. Through the production of paraffin sections and antigen retrieval, tissue sections were blocked using 5\% BSA for $1 \mathrm{~h}$. Then, cells and sections were incubated using primary against CD29 (sc-9970, 1:200, Santa Cruz), CD34 (bs-0646R, 1:500, bioss), CD44 (bs-4916R, 1:400, bioss), CD45 (bs-0522R, 1:300, bioss),
CD90 (bs-0778R, 1:500, bioss), nephrin (sc-377246, 1:100, Santa Cruz), LC3 (12135-1-AP, 1:200, Proteintech) at $4{ }^{\circ} \mathrm{C}$ overnight. After incubation with secondary antibodies at $37^{\circ} \mathrm{C}$ for $1 \mathrm{~h}$, samples or cells were stained with DAPI for 5 min and laser confocal microscopy was adopted for observation and photo taking.

\section{Flow cytometry}

ADSCs in passage 3 were digested by $0.25 \%$ trypsin, and cell suspension were filtered using 100-mesh filter. After 5 min of centrifugation, supernatant was removed and cells were resuspended with PBS followed by incubating with primary antibodies. Positive marker of ADSCs were including CD29 (1:500), CD44 (1:300), and CD90 (1:500). CD34 (1:300) and CD45 (1:300) were the negative marker of ADSCs. For exosome, the exosome suspension was used directly for purity identification by FACS according to previous study [20]. Positive marker of exosome, CD9 (bs-2489R, 1:100, Bioss), CD63 (GTX41877, 1:100, GeneTex), and CD81 (GTX41794, 1:100, GeneTex) were used for purity identification. In brief, exosomes were resuspended in $200 \mu \mathrm{L}$ of PBS and aldehyde/sulfate beads ( $10 \mu \mathrm{L}$, Life Technologies) were added into exosome solution. After mixing for $15 \mathrm{~min}$ at room temperature by using a benchtop rotator, $600 \mu \mathrm{L}$ PBS were added and incubated overnight at $4{ }^{\circ} \mathrm{C}$. The next day, the pellets were resuspended in $40 \mu \mathrm{L}$ of $2 \%$ BSA in PBS and stained with CD9, CD63, CD81, and their isotype control antibodies at RT for $30 \mathrm{~min}$. After centrifugation at $12,000 \mathrm{rpm}$ for $1 \mathrm{~min}$, the pellets were resuspended in $20 \mu \mathrm{L}$ BSA and stained with secondary antibodies. Eventually, the beads containing exosome were analyzed using the LSR Fortessa X-20 cell analyzer (BD Biosciences).

For apoptosis detection, cells were digested using $0.25 \%$ trypsin without EDTA and centrifuged at 1500 $\mathrm{rpm}$ for $5 \mathrm{~min}$. The precipitate was resuspended and washed gently with PBS. Through 5 min of centrifugation at $1500 \mathrm{rpm}$, cells were suspended using $300 \mu \mathrm{L}$ binding buffer. Cells were stained with $5 \mu \mathrm{L}$ Annexin V-FITC in darkness for $5 \mathrm{~min}$ following with $10 \mu \mathrm{L}$ PI staining for $10 \mathrm{~min}$ at room temperature. Then, cells apoptosis was measured by Flow cytometry (BD Biosciences, FACSCalibur).

\section{CCK8 assay and Tunel staining}

MPC5 cells treated with high-glucose or ADSCs-Exo were seeded on 96-well plate at a density of 3000 cells/ well. After $24 \mathrm{~h}, 48 \mathrm{~h}, 72 \mathrm{~h}$, and $96 \mathrm{~h}$ of incubation, $100 \mu \mathrm{L}$ CCK8 was added into cells following by $2 \mathrm{~h}$ of incubation. OD value was detected through microplate reader (Thermo, Multiskan MK3) at the wavelength of $450 \mathrm{~nm}$. 
For tissue apoptosis measurement, tissue sections were assessed according to the manufacturer's instruction (Biobox, BA27). In brief, the specimens were stand in TUNEL reaction solution containing $\mathrm{TdT}$ and Streptavidin-FITC to label DNA fragments. After washing, the labeled preparations were incubated using POD-conjugated Anti-FITC at $37{ }^{\circ} \mathrm{C}$ for $30 \mathrm{~min}$ in darkness. Then, slides were added with $\mathrm{DAB}$ reaction solution and stained with DAPI followed by a step of hydrochloric acid alcohol differentiation, ethanol dehydration, xylol incubation, and mounting with neutral resins. The resulting images were processed and analyzed using microscope.

\section{RNA isolation and quantitative real-time polymerase chain reaction}

Tissue and cell total RNA were extracted using TRIZOL reagent. One microgram RNA was used to perform reverse transcription by using First Strand cDNA Synthesis Kit (Fermentas, K1622) and polymerase chain reaction using SYBR Green qPCR kit (Thermo, K0222). Primers of miR-486 and internal control were as follows: miR486, TATAGCTCCTGTACTGAGCTGC; miR universal primer, CCAGTCTCAGGGTCCGAGGTATTC. U6 F, CTCGCTTCGGCAGCACA; U6 R, AACG CTTCACGAATTTGCGT.

\section{Western blotting}

Protein of renal tissue and MPC5 cells were processed SDS-PAGE according to the previous study [21]. All primary antibodies were included CD9 (1:1000, Bioss, bs-2489R), CD63(1:1000, GeneTex, GTX41877), CD81 (1:800, GeneTex, GTX41794), $\beta$-actin (1:1000, CST, 4970), Caspase3 (1:1000, CST, 9662), mTOR (1:1000, CST, 2983), p-mTOR (1:500, CST, 5536), LC3 (1:500, Proteintech, 12135-1-AP), p62 (1:500, Proteintech, 18420-1-AP), Beclin1 (1:500, CST, 3495) and Smad1 (1:1000, Affinity, AF6451).

\section{Statistical analysis}

Statistical analysis was processed by utilizing SPSS 21.0 software. Data were presented according to three independent experiments and appeared as mean \pm standard error of the mean. Histogram and line chart were analyzed by GraphPad Prism 5.0 (GraphPad Software Inc., USA) using Student's $t$ test or One-way ANOVA analysis. $p<0.05$ was regarded as significant difference.

\section{Results}

Adipose-derived stem cells (ADSCs) possesses strong differentiation capability

In order to obtain ADSCs-derived exosome, ADSCs were isolated from subcutaneous adipose tissue in groin of $\mathrm{C} 57 \mathrm{BL} / \mathrm{Ks} \mathrm{Jb} / \mathrm{m}$ mice. At passage 3 of ADSCs, cell purity was assessed by performing IF staining using positive marker (including CD29, CD44 and CD90) and negative marker (including CD34 and CD45) of ADSCs. As shown in Additional file 1: Figure S1A, most of ADSCs expressed CD29 (red), CD44 (green), and CD90 (red) but not CD34 and CD45 (Additional file 1: Figure $\mathrm{S} 1 \mathrm{~A})$. A relatively greater number of CD29-, CD44-, and CD90-positive cells were observed by flow cytometry in ADSCs. However, there are no CD34- and CD45-positive cells in ADSCs (Additional file 1: Figure S1B). Through performing flow cytometry and CCK8 assays, we determined that passage 6 of ADSCs had strong growth capacity (Additional file 1: Figure S2A-C). To confirm the differentiation ability of ADSCs, cells were cultured with osteogenic- and adipogenic medium. Next, ALP activity detection and Alizarin Red Staining results were the indicators of osteogenic capability of ADSCs which showed that osteogenic medium significantly induced the level of ALP and the number of Alizarin Red staining positive cells in ADSCs (Additional file 1: Figure S2D-E). Oil Red O staining results showed higher adipogenic potential under the condition of adipogenic medium (Additional file 1: Figure S2F). Thus, these data indicate that the ADSCs are isolated successfully.

\section{Adipose-derived stem cells are efficient producers of exosome}

Extracellular vesicles (EV), term used as the exosome characteristics, were isolated from ADSCs to explore the role of ADSCs-Exo on podocyte damage. Firstly, we identified the purity of ADSCs-Exo by flow cytometry using immune-labelling method. The results showed that $89.3 \%$ of CD9-, $94.6 \%$ of CD63-, and $93.7 \%$ of CD81-positive particles in the isolated EV (Fig. 1a). Exosome surface markers CD9, CD63, and CD81 were also presented high expression in comparison with ADSCs (Fig. 1b). Through the observation using transmission electron microscopy, ADSCs-Exo appeared as circular particles (Fig. 1c). With NTA analysis, the size distribution of total extractive focused on the average size of $93.24 \mathrm{~nm}$ which was in accord with the feature of exosome $(40-120 \mathrm{~nm})$ (Fig. 1d). The total particles of ADSCs-exosome was found to be high, averaging around $1.73 \times 10^{8} / \mathrm{mL}$ (Fig. 1d). Collectively, we successfully obtain exosome particles from ADSCs.

\section{ADSCs-Exo significantly attenuates pathologic symptom and cell apoptosis of spontaneous diabetes mice}

Since USCs-derived exosome possesses protection effect on renal injury and podocyte apoptosis in rat, we try to explore whether ADSCs-Exo also have the same role as well as USCs-Exo. Firstly, in spontaneous diabetes mice, we found that levels of urine protein within $24 \mathrm{~h}$ (two-fold), Scr (three-fold), and BUN (three-fold) were robustly 


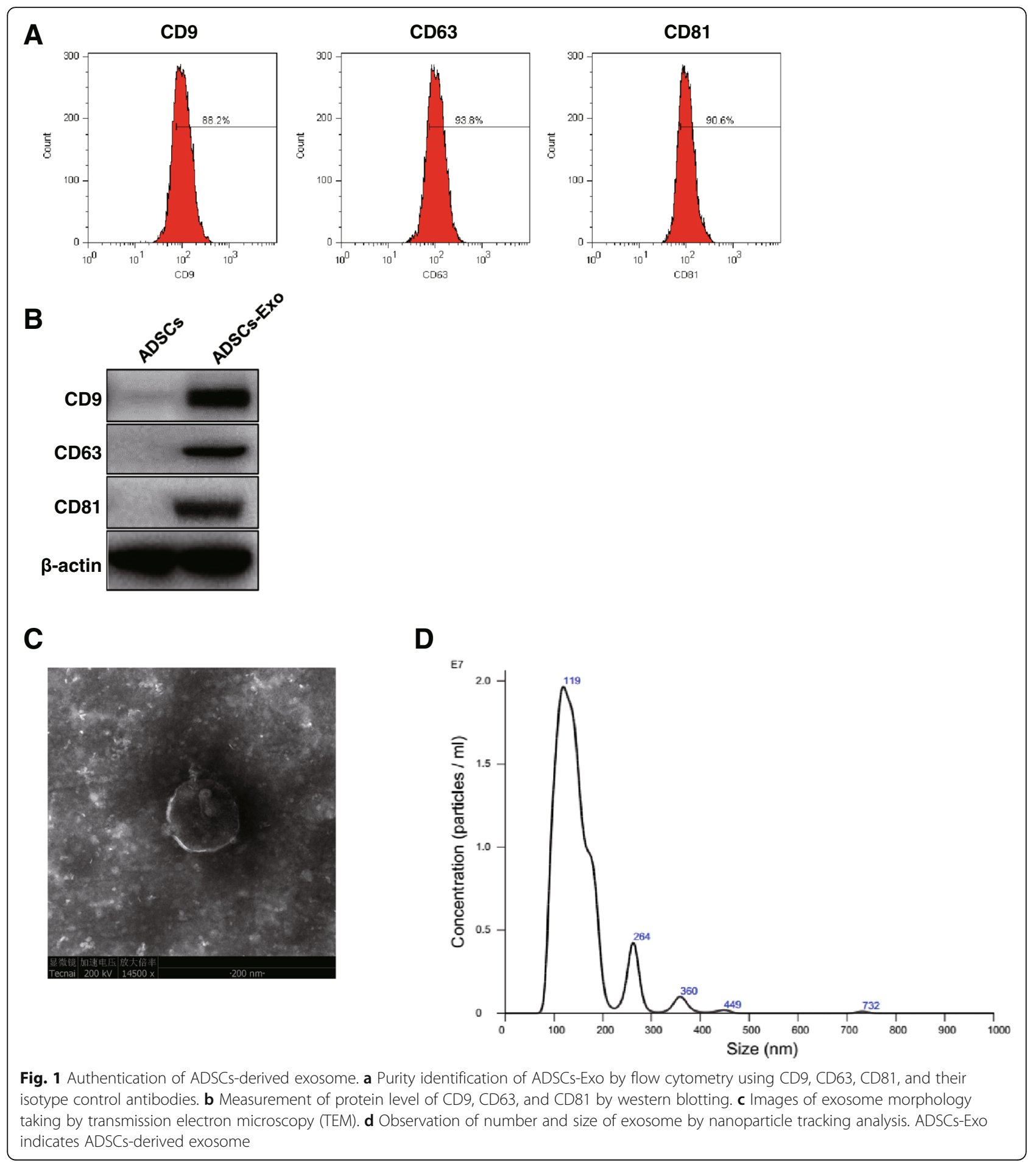

enhanced compared to $\mathrm{C} 57 \mathrm{BL} / \mathrm{Ks}$ ) $\mathrm{db} / \mathrm{m}$ (control group) (Additional file 1: Figure S3A-C). Besides, PAS staining results suggested that renal tissue had presented glomerular extracellular matrix (ECM) accumulation, glomerular mesangial cell proliferation, and base-membrane thickness in spontaneous diabetes mice compared to control mice indicating $\mathrm{DN}$ mice model was successfully established (Additional file 1: Figure S3D). Once administration with ADSC-Exo according to the flow chart in Additional file 1: Figure S3E, the increase of urine protein, Scr, and BUN were inhibited observably (Fig. 2a-c). Additionally, ADSCsExo administration significantly attenuated pathological changes in DN mice model (Fig. $2 \mathrm{~d}$, the right 
A

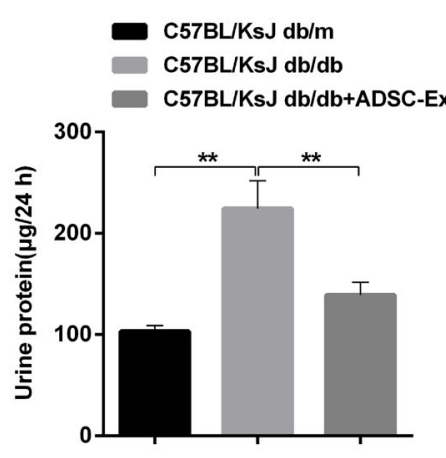

D

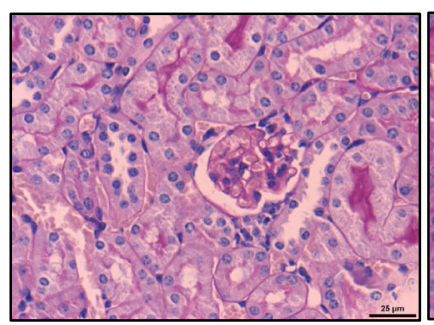

C57BL/KsJ db/m
B

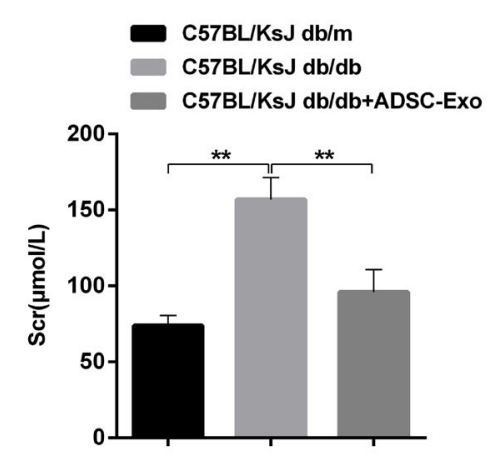

\section{C}

C57BL/KsJ db/m

C57BL/KsJ db/db

C57BL/KsJ db/db+ADSC-Exo

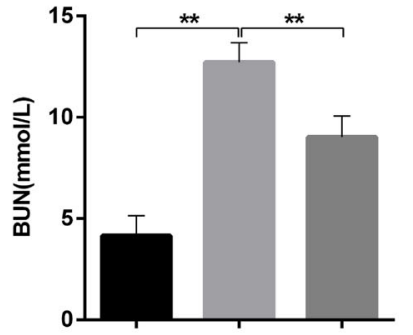

E

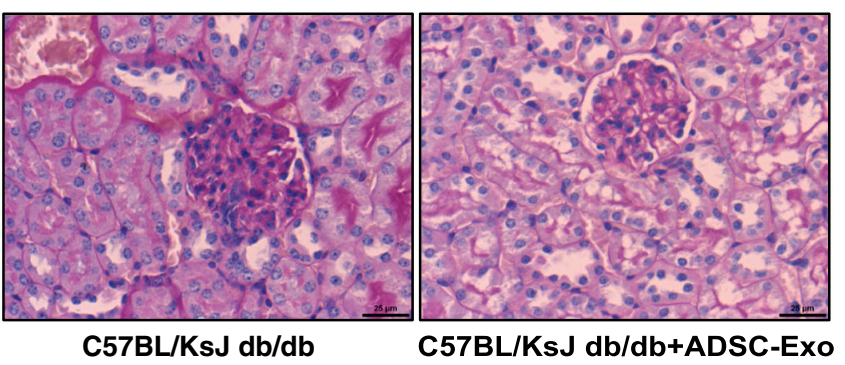

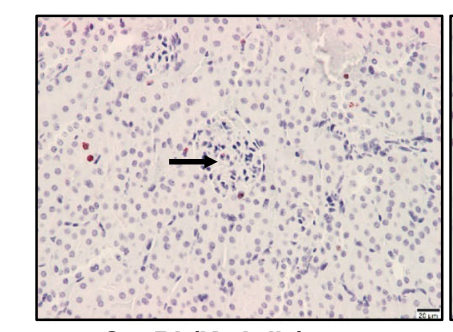

C57BL/KsJ db/m

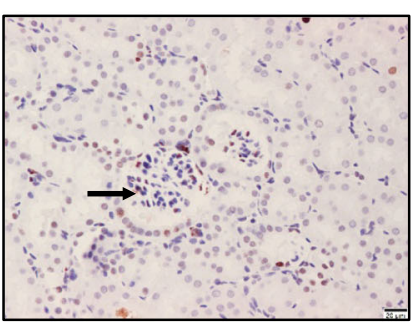

C57BL/KsJ db/db
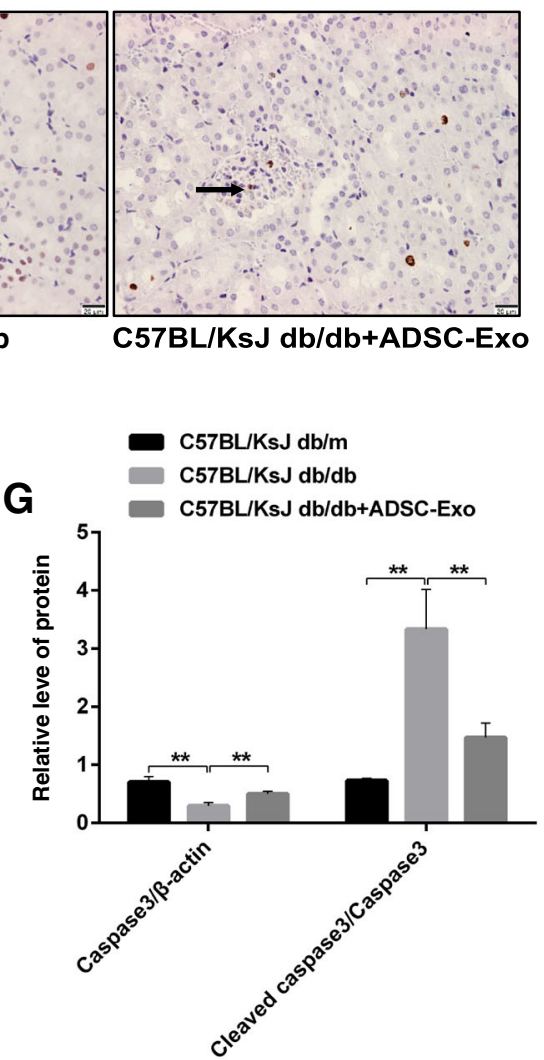

Fig. 2 Effects of ADSCs-Exo on spontaneous diabetes mice. a-c Measurement of urine protein, Scr, and BUN levels in control and spontaneous diabetes mice before and after ADSCs-Exo treatment. $\mathbf{d}$ PAS staining of renal tissue section in three group mice. e Detection of apoptotic cells by Tunel staining in renal specimen. Black arrow indicates apoptotic cells. Brown color indicates positive cells. $\mathbf{f}$ Expression examination of caspase3 and cleaved-caspase3 in renal tissue by western blotting. $\mathbf{g}$ Relative quantitative analysis of caspase3 and cleaved-caspase3. Scale bar, $25 \mu \mathrm{m} .{ }^{* *} p<0.01$ 
A
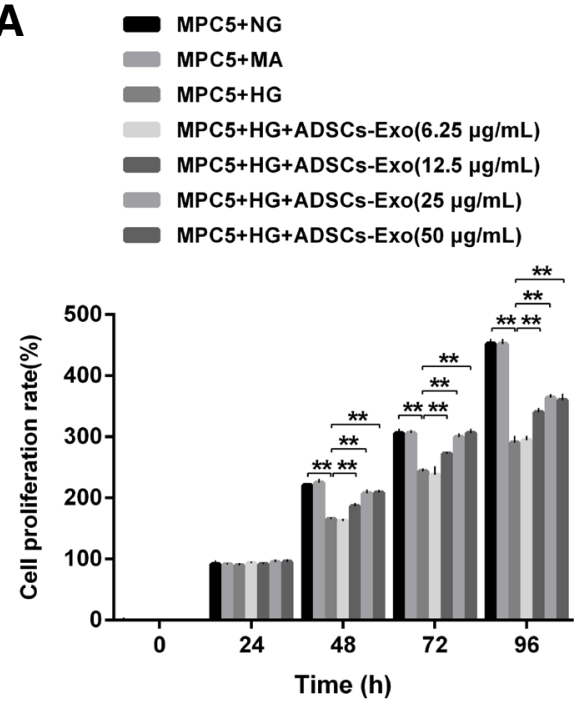

D

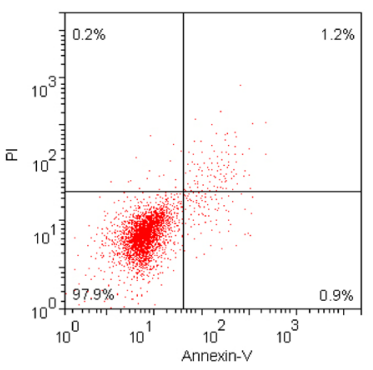

MPC5+NG
B

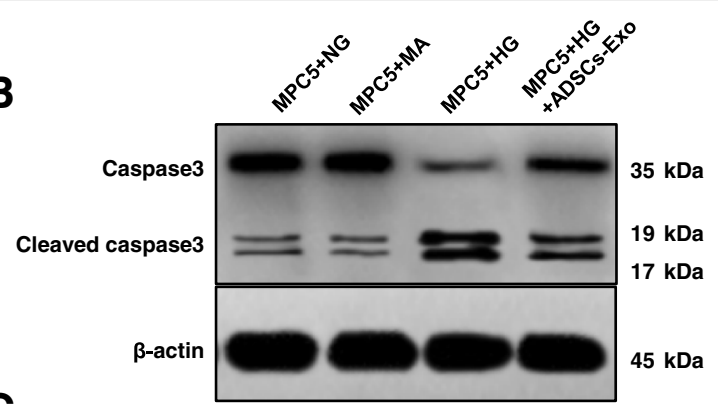

C

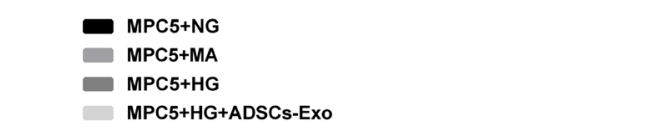

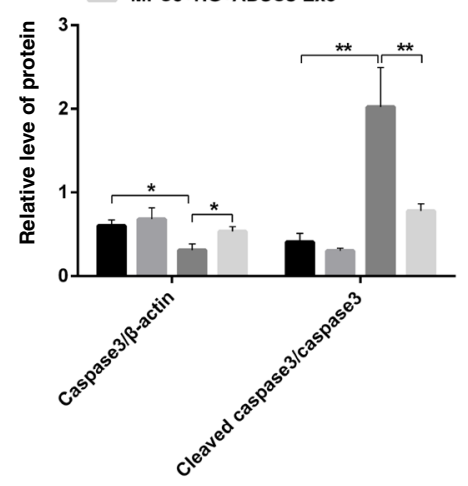

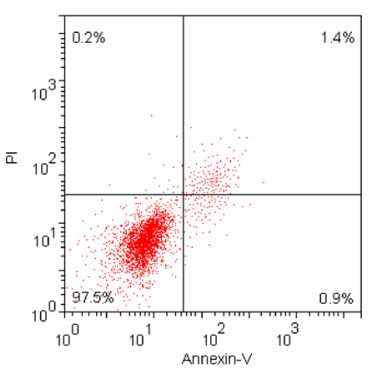

MPC5+MA

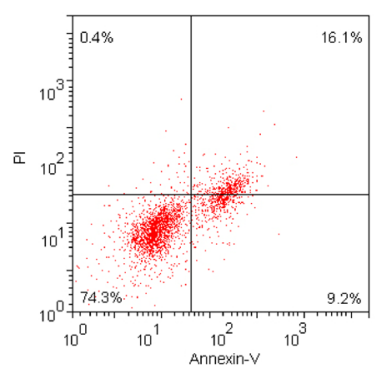

MPC5+HG

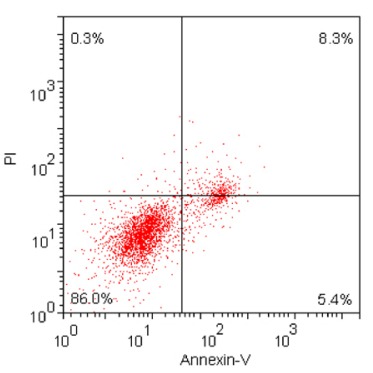

MPC5+HG+ADSCs-Exo

E

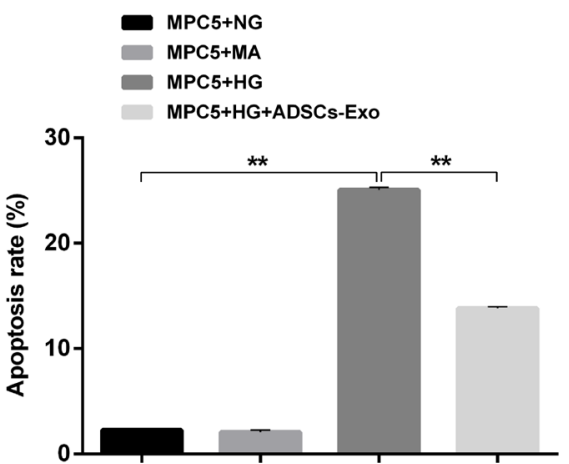

Fig. 3 Impacts of ADSCs-EXo on high glucose-induced MPC5 cells apoptosis. a MPC5 cells viability was evaluated through CCK8 assay under the condition of normal glucose (NG), mannitol (MA), high glucose (HG), and concentration gradient ADSCs-Exo for 24 h, 48 h, 72 h, and 96 h. b Expression examination of caspase 3 and cleaved-caspase 3 in MPC5 cells by western blotting. $\mathbf{c}$ Relative quantitative analysis of caspase 3 and cleaved-caspase3 in NG/MA/HG/ADSCs-Exo treated MPC5 cells. d Apoptotic MPC5 cells were determined by flow cytometry. e Relative apoptosis rate of MPC5 cells under the treatment of NG/MA/HG/ADSCs-Exo. NG indicates $5.5 \mathrm{mM}$ D-glucose. MA indicates $5.5 \mathrm{mM}$ D-glucose+ $24.5 \mathrm{mM}$ mannitol. HG indicates $30 \mathrm{mM}$ D-glucose. ${ }^{* *} p<0.01$ 


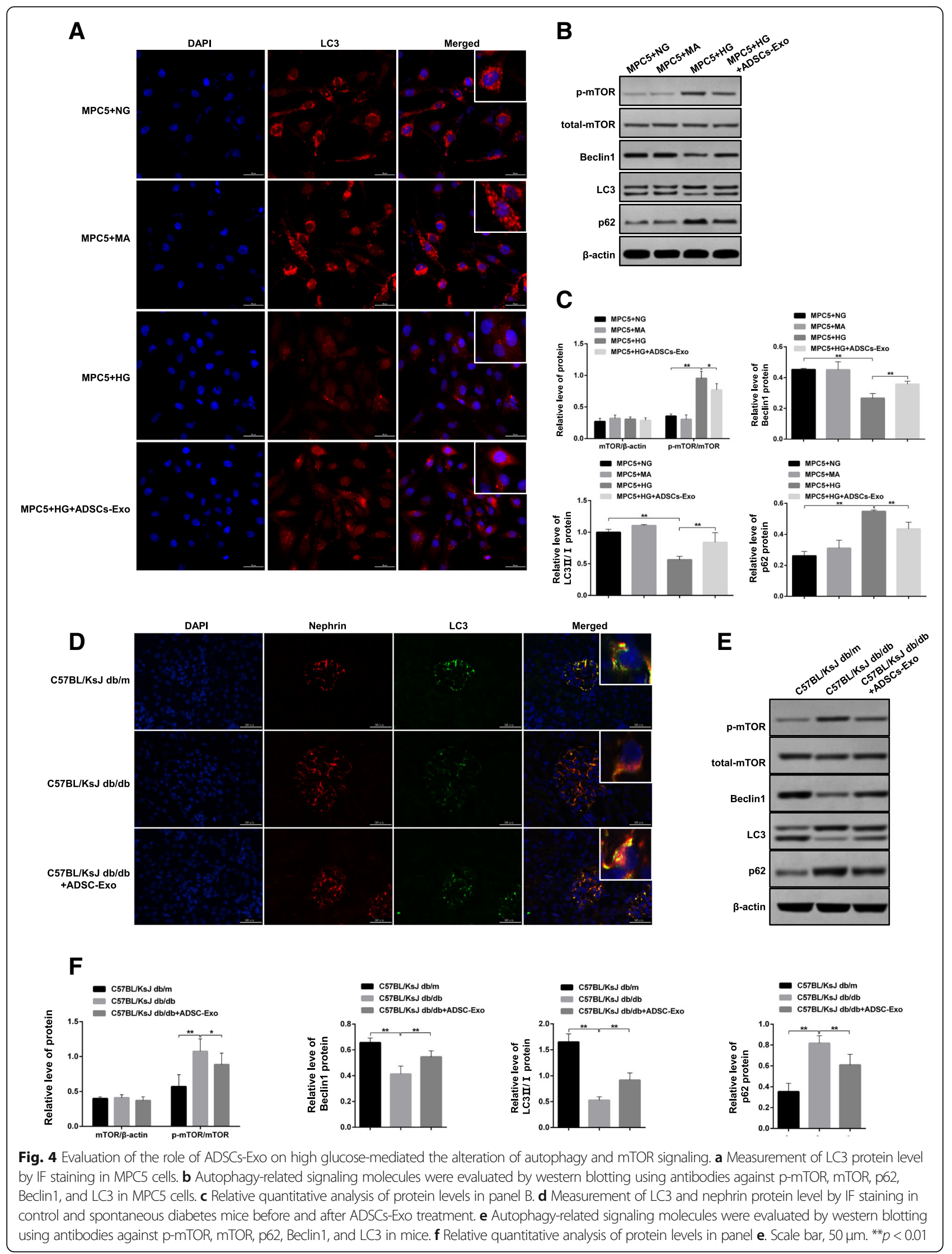




\section{A}
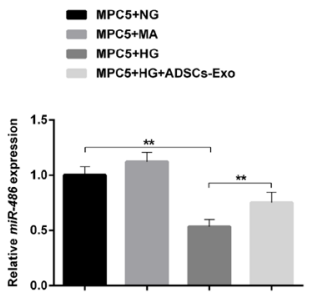

D

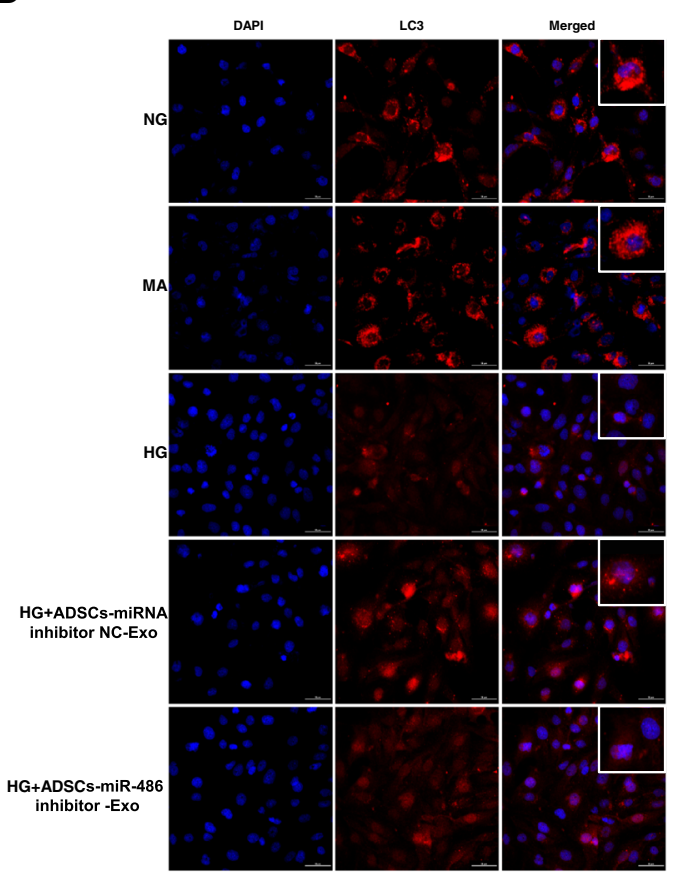

B

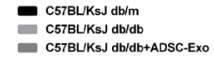

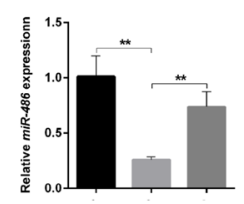

C

$\mathrm{Z}_{\mathrm{MB}}^{\mathrm{NG}}$

HG+ADSCs-miRNA inhibitor NC.Exo

- HG+ADSCs-miR-486 inhibitor-Exo

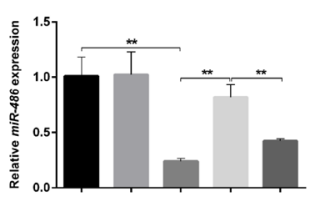

E

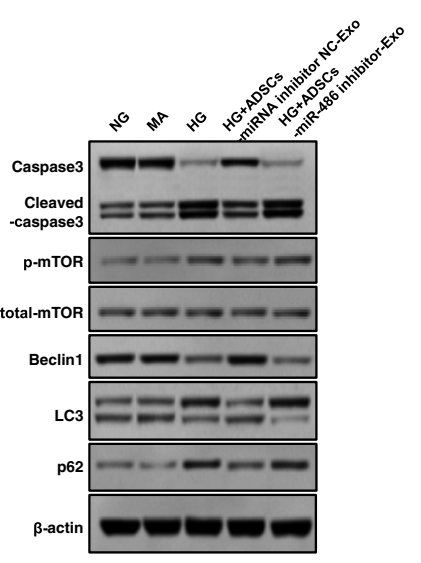

$\mathbf{F}$

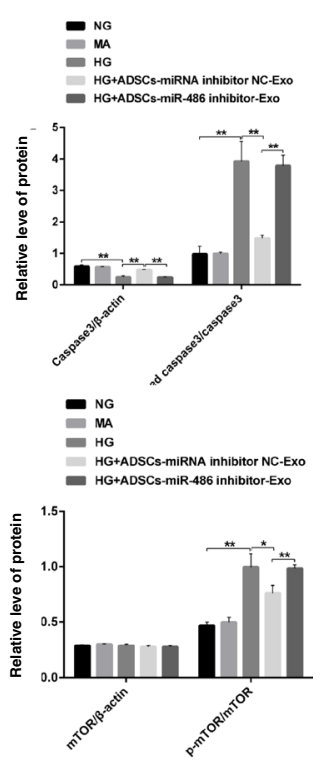

를

HG

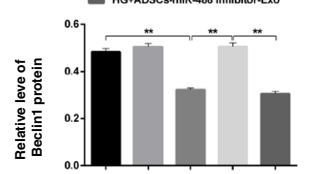

H

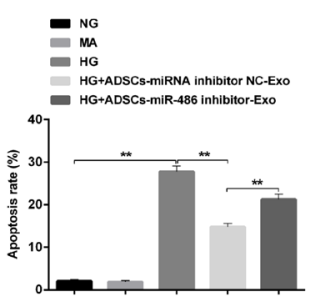

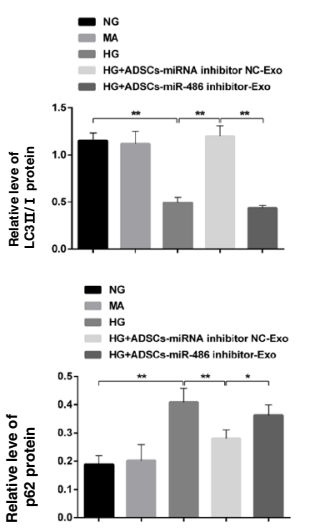

Fig. 5 Exploration of the role of miR-486 on ADSCs-Exo-mediated damage inhibition in MPC5 cells. $\mathbf{a}, \mathbf{b}$ Expression examination of miR-486 in ADSCs-Exo-treated MPC5 cells and renal tissue by qRT-PCR. $\mathbf{c}$ miR-486 expression was evaluated by qRT-PCR in HG-induced MPC5 cells treated with ADSCs-Exo NC and ADSCs-Exo-miR-486 inhibitor. d Measurement of LC3 protein level by IF staining in MPC5 cells treated with HG/MA/HG/ ADSCs-Exo/ADSCs-Exo-miR-486 inhibitor. e Apoptosis- and autophagy-related molecules were determined by western blotting using primary antibodies against caspase3, cleaved-caspase3, p-mTOR, mTOR, p62, Beclin1, and LC3 in MPC5 cells. f Relative quantitative analysis of protein levels in panel e. $\mathbf{g}$ Apoptotic MPC5 cells were determined by flow cytometry under conditional treatment. $\mathbf{h}$ Relative apoptosis rate of MPC5 cells in panel g. Scale bar, $50 \mu \mathrm{m} .{ }^{*} p<0.05 ;{ }^{* *} p<0.01$ 
A

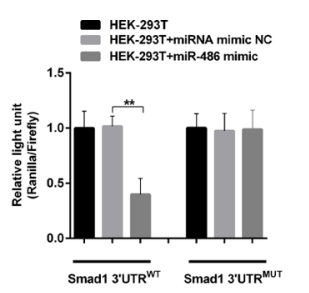

B
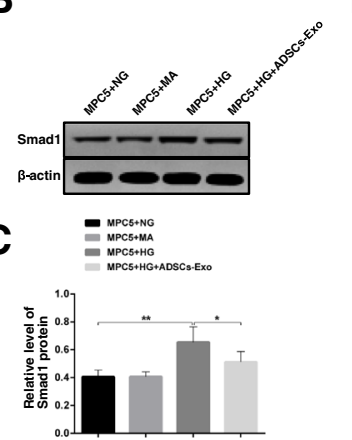

D

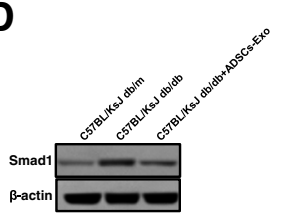

E
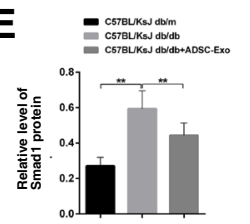

$\mathbf{F}$

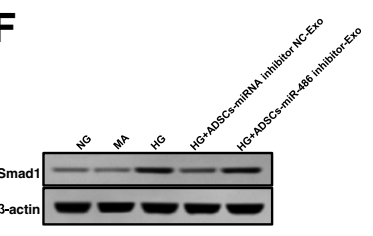

G

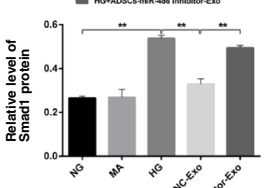

H

I
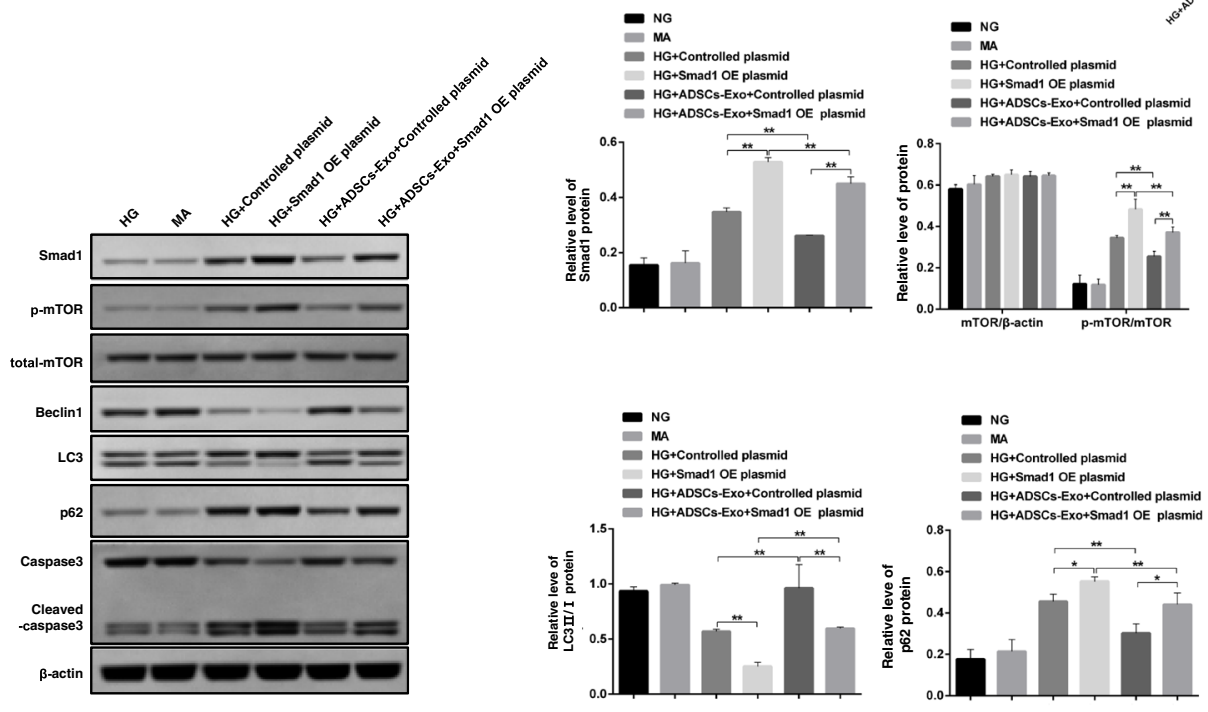

\section{- MA}

- $\begin{aligned} & \mathrm{HG}+\text { Controlled plasmid } \\ & \mathrm{HG}+\text { Smad1 OE plasmid }\end{aligned}$

HG+Smad1 OE plasmid
HG+ADSSs-Exo+Controlled plasmid

HG+ADSCs-Exo+Controlled plasmid
HG+ADSCs-Exo+Smad1 OE plasmid
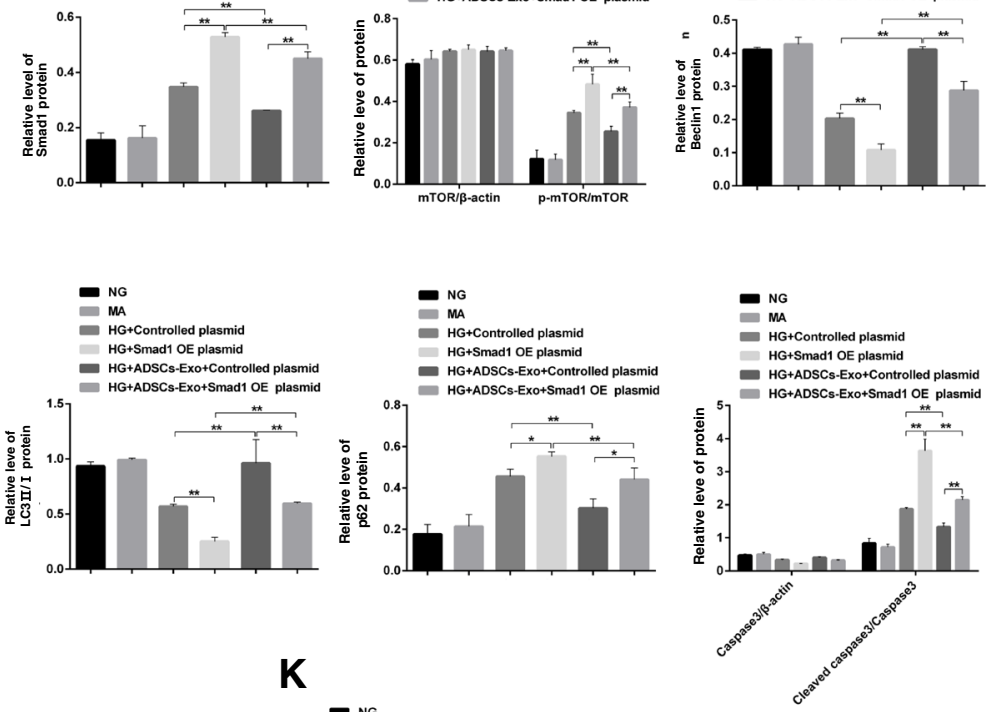

J

K

른 ${ }_{\text {MA }}^{\text {Ma }}$

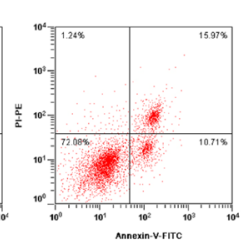

$\mathrm{HG}+\mathrm{C}$ Conontronterfed plasmid
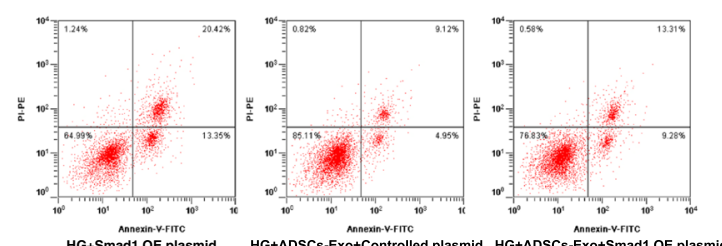

Fig. 6 Mechanism exploration of ADSCs-Exo-mediated injury protection in DN and high glucose-induced apoptosis in MPC5 cells. a Luciferase activity of Smad1 was measured by utilizing dual luciferase reporter assay system. b, c Protein and relative quantitative analysis of Smad1 expression in MPC5 cells treated with NG, MA, HG, and ADSCs-Exo. d, e Protein and relative quantitative analysis of Smad1 expression in control and spontaneous diabetes mice before and after ADSCs-Exo treatment. $\mathbf{f}, \mathbf{g}$ Protein and relative quantitative analysis of Smad1 expression in MPC5 cells treated with NG, MA, HG, ADSCs-Exo-NC, and ADSCs-Exo-miR-486 inhibitor. $\mathbf{h}$ Protein levels of Smad1, caspase3, cleaved-caspase3, p-mTOR, mTOR, p62, Beclin1, and LC3 in MPC5 cells treated with NG, MA, HG-NC, HG-Smad1, ADSCs-EXo, and ADSCs-Exo-Smad1. i Relative quantitative analysis of protein expression in panel $\mathbf{h}$. $\mathbf{j}$ Apoptotic MPC5 cells were determined by flow cytometry. $\mathbf{k}$ Relative apoptosis rate of MPC5 cells in panel $\mathbf{j}$. ${ }^{*} p<0.05 ;{ }^{* *} p<0.01$ 
image vs the middle image). It is confirmed that podocyte apoptosis is the hallmark of DN. As you saw from our tunel staining result, the increase of cell apoptosis in $\mathrm{C} 57 \mathrm{BL} / \mathrm{Ks} \mathrm{db} / \mathrm{db}$ was remarkably reduced under the treatment with ADSCs-Exo (Fig. 2e, the right image vs the middle image). Apoptotic signal protein such as cleaved caspase 3 was promoted in DN mice, whereas suppressed in the present of ADSCs-Exo (Fig. 2f-g). These results demonstrate that ADSCs-Exo can relieve the pathology symptom in DN mice.

\section{ADSCs-Exo effectively inhibits high glucose-induced cell apoptosis in MPC5 cells}

The protection effect of ADSCs-Exo on podocyte damage was not only found in DN mice. In mouse podocyte MPC5 cells, high glucose induced the cell growth inhibition compared in the control group (NG- and MA-treated cells). ADSCs-Exo treatment effectively relieved the negative effect of high glucose on MPC5 cells in concentration- and time-dependent manners $(\leq 25 \mu \mathrm{g} /$ $\mathrm{mL}$ ), especially in the concentration of $25 \mu \mathrm{g} / \mathrm{mL}$ at $48 \mathrm{~h}$ (Fig. 3a). Through immunoblotting experiment, we found that $25 \mu \mathrm{g} / \mathrm{mL}$ of ADSCs-Exo vividly reduced the activity of cleaved caspase 3 which showed high level under the condition of high glucose (Fig. 3b, c). In the analysis results of flow cytometry, high glucose-mediated the increase of apoptotic cell proportion was sharply reduced with the treatment of ADSCs-Exo (Fig. 3d, 16.1+ $9.2 \%$ vs $8.3+5.4 \%$ ). Combined with the statistical result of apoptosis rate in Fig. 4e (Fig. 3e), we conclude that ADSCs-Exo also ameliorate high glucose-induced podocyte injury in vitro.

\section{Autophagy induction and mTOR signaling activation in high glucose-induced MPC5 cells and DN mice are suppressed by ADSCs-Exo treatment}

Autophagy dysfunction always is the indicator of podocyte apoptosis/damage in vitro and in vivo [22]. Due to the improvement role of ADSCs-Exo on cell apoptosis in MPC5 cells and DN mice, whether ADSCs-Exo-mediated the protection effect was involved in the process of autophagy downregulation-induced cell apoptosis remained unclear and needed further investigation. LC3 was the common indicator protein of autophagosome. Once autophagy was downregulated, the rate of LC3 II/LC3 I was repressed which was contributed to the process of podocyte apoptosis. In the IF staining assay, high glucose obviously decreased the description of point aggregate of LC3 protein indicating autophagy reduction was involved in the process of HG-mediated cell apoptosis in MPC5 cells. But ADSCs-Exo administration significantly recovered the decrease of LC3 level (Fig. 4a). Numerous studies have confirmed that mTOR signaling and p62 level were the key regulator of autophagy in DN. After performing western blotting assay, we discovered that both phosphorylation of mTOR and p62 level were enhanced under the condition of high glucose followed by the decrease of the marker protein of autophagy such as Beclin1 and LC3II/I. Conversely, treatment with ADSCs-Exo inhibited mTOR and p62/LC3 signaling pathway and enhanced the expression of autophagy-related proteins (Fig. 4b, c). In DN mice, expression of LC3 in podocyte which was positively stained with nephrin was reduced obviously (Fig. 4d). Besides, increase of mTOR and p62/LC3 signaling transduction and decrease of autophagy marker expression were also observed in DN mice (Fig. 4e). However, ADSCs-Exo injection remarkably restored these changes which suggested that the restoration of autophagy was required in ADSCs-Exo-mediated the improvement of podocyte damage in MPC5 cells and DN mice (Fig. 4d, f).

\section{miR-486 is required in the process of ADSCs-Exo- mediated the injury protection in MPC5 cells}

In comparison to normal people, diabetic patients showed lower expression of miR-486 implying that miR-486 might be a critical regulator in the process of DN. To investigate the molecule mechanism of ADSCsExo-launched damage protection, we determined the expression pattern of miR-486 under the treatment with and without ADSCs-Exo. We found that the level of miR-486 was significantly downregulated in high glucose-induced MPC5 cells and DN mice, whereas the expression of miR-486 was recovered in the present of ADSCs-Exo in vitro and in vivo (Fig. 5a, b). These results motivated us to speculate whether miR-486 of ADSCs-Exo could affect high glucose-induced MPC5 cells and renal injury. Firstly, we verified the inhibition effect of miR-486 inhibitor. As shown in Additional file 1: Figure S4, miR-486 inhibitor transfection significantly repressed the level of miR-486 in ADSCs and ADSCsExo (Additional file 1: Figure S4A-C). Once the inhibition of the expression of miR-486 in ADSCs using miR-486 inhibitor, ADSCs-Exo-induced upregulation of miR-486 in the presence of HG was limited observably (Fig. 5c). To further explore the role of miR-486 on autophagy downregulation-mediated podocyte apoptosis in MPC5 cells, we evaluated the expression of autophagyand apoptosis-related proteins in the present of miR-486 inhibitor. As you could see, ADSCs-Exo restored high glucose-induced decrease of LC3 level, but miR-486 inhibition in ADSCs dramatically blocked the protection role of ADSCs-Exo on LC3 expression in MPC5 cells (Fig. 5d). Under the same conditions, ADSCs-Exo mediated the upregulation of autophagy, including reducing high glucose-induced upregulation of mTOR and p62 signaling and the downregulation of Beclin1 and LC3II/I, was thoroughly suppressed through regulating the signaling 
molecules mentioned above (Fig. 5e-f). Besides autophagy, ADSCs-Exo-launched protective effect on cell damage was also neutralized in the presence of miR-486 inhibitor. For example, cleaved caspase3 showed the same level with HG-treated group in miR-486 inhibitor group indicating miR-486 inhibition in ADSCs which fully reversed the role of normal ADSCs-Exo (Fig. 5e, f). By performing flow cytometry, we also discovered that ADSCs-Exo mediated the decrease rate of apoptotic cells which weakened in the condition of miR-486 inhibition (Fig. 5g, h). These data indicate that miR-486 in ADSCs was needed in the course of ADSCs-Exo-mediated protective effect on cell injury through regulating autophagy-related signaling pathway.

\section{ADSCs-Exo ameliorates podocyte damage by regulating miR-486/Smad1/mTOR signaling pathway}

To explore the direct target of miR-486 in MPC5 cells, we screened the target gene of miR-486 using the online prediction software and found that miR-486 could directly bind to smad1's 3'UTR region implying smad1 might be the specific target gene of miR-486 in MPC5 cells. Combined with dual luciferase reporter assay, we discovered that the luciferase reporter activity of smad1 $3^{\prime} \mathrm{UTR}^{\mathrm{WT}}$ was obviously inhibited in the group of miR-486 mimic-treated cells compared to miRNA mimic NC group. However, miR-486 mimic had no inhibition effect on cells transfected with smad1 $3^{\prime} \mathrm{UTR}^{\mathrm{MUT}}$ indicating smad1 was indeed the target gene of miR-486 (Fig. 6a). Besides, we also discovered that smad1 was upregulated in high glucose-induced MPC5 cells and DN mice. But ADSCs-Exo administration fully recovered the expression of smad1 in vitro and in vivo (Fig. 6b-e). Importantly, ADSCs-Exo derived from ADSCs treated with miR-486 inhibitor showed the same expression quantity of smad1 implying miR-486/smad1 signaling pathway was involved in ADSCs-Exo-launched injury repair (Fig. 6f, g). Based on these, we established the overexpressed construct of smad1 to further illustrate the role of miR-486/smad1 signaling on ADSCs-Exo-mediated damage repair. The overexpressed efficiency was verified through western blotting assay (Additional file 1: Figure S5A-B). It is confirmed that smad1 participates in the mTOR signaling pathway in many cases [23, 24]. In our study, we confirmed that HG induced the activation of mTOR/p62 signaling pathway and cleaved caspase3 expression; the inhibition of Beclin1 and LC3II/I were enlarged when smad1 was overexpressed in MPC5 cells (Fig. 6h). Though ADSCs-Exo could reverse these changes of signaling molecules by downregulating smad1, ADSCs-Exo had no effect on these molecules when smad1 were overexpressed in MPC5 cells (Fig. 6h, i). HG-induced apoptotic rate was attenuated by ADSCs-Exo treatment, but smad1 overexpression blunted the role of ADSCs-Exo
(Fig. 6j, k). In a word, miR-486/smad1/mTOR might be the main signaling pathway in the process of ADSCs-Exo-mediated damage repair in MPC5 cells and DN mice.

\section{Discussion}

Diabetic nephropathy as a serious microvascular complication of both type 1 and type 2 diabetes mellitus is expected to contribute to a significant percentage of the worldwide morbidity rate $[25,26]$. Therefore, there is urgency to investigate novel therapeutic target to combat diabetic nephropathy. In the present study, we confirmed that ADSCs-Exo significantly prevented from renal injury by regulating autophagy homeostasis in podocyte. Maybe, ADSCs-Exo would be a new therapeutic target for DN treatment.

Transient presence of MSCs can accelerate the process of renal repair in mice through providing a paracrine support including the secretion of exosomes, growth factor, and cytokines $[27,28]$. The evidence is mounting that exosomes derived from ADSCs act the core effects on protecting podocyte from conditioned medium-mediated damage [29, 30]. ADSCs-Exo overexpressing nuclear factor-E2-related factor 2 (Nrf2) promote wound healing and can be suitable for clinical application in the treatment of diabetic foot ulcers (DFU) [31]. In our research, we verified that ADSCs-Exo administration prevented renal injury from diabetes and the protective role of ADSCs was mainly based on its paracrine.

Podocytopathy such as podocyte damage has been regarded as the research focus in deciphering cellular and molecular mechanisms of DN [32]. Dysregulated cellular homeostasis is the hallmark of podocyte injury in DN. As catabolic process, autophagy dysfunction-mediated imbalance of homeostasis has been suggested to play pathogenic roles in podocyte/renal injuries in DN mice model with involving in many signal transduction pathways [33, 34]. Hyperactivation of the mTOR pathway in DN plays a pivotal role in the process of podocyte injury and the decline of glomerular filtration rates [35]. Damaged podocyte is always accompanied by the decrease of autophagy flux, the accumulation of p62, and the interaction between p62 and LC3 [36]. Therefore, mTOR and p62/LC3 signaling transduction are involved in the pathogenesis of autophagy dysfunction-mediated podocyte injury. Administration with ADSCs-Exo, activation of mTOR, and enhancement of p62 expression and LC3 II/LC3 I rate in high glucose-exposed MPC5 cells and DN mice were significantly inhibited indicating ADSCs-Exo-mediated increase of autophagy flux in damaged podocyte.

Stem cells-derived exosomes are able to transport bioactivators between cells through a new mechanism of cell-to-cell communication, including growth factors, mRNA, and miRNA [27, 37]. Based on this feature, exosomes have got a lot of attention with respect to drug 
delivery. MSC exosomes enriched in miR-146b can decrease tumor size in glioblastoma multiforme (GBM) by intratumoral injection [38]. Exosomes secreted from MSCs can also transfer miR-143 to GBM cells leading to migration and self-renewal inhibition [39]. Recently, microRNAs (miRNAs) in the extracellular environment have emerged as critical regulators in renal fibrosis, acute kidney injury, $\mathrm{DN}$, and progressive kidney disease [40-42]. Especially, miRNAs packaged in extracellular vesicles such as exosomes showed concentration changes associated with DN occurrence and received significant attention as potential noninvasive biomarkers for diagnosis and treatment of DN [43]. In our study, miR-486 enriched in ADSCs-Exo induced the upregulation of miR-486 in MPC5 cells and DN mice. miR-486 inhibition in ADSCs significantly suppressed the protection role of ADSCs-derived exosome in high glucose-induced podocyte damage and autophagy reduction. Eventually, we confirmed that miR-486 could directly regulate Smad1 expression followed by the increase of mTOR-mediated autophagy flux. Our data demonstrated that ADSCs-Exo mediated the transport of miR-486 to podocyte and the upregulation of miR-486 suppressed renal injury by regulating smad1/mTOR signaling pathway.

\section{Conclusions}

In conclusion, miR-486 carried by ADSCs-Exo could transfer to podocyte functioning as an activator of autophagy and relieve cell damage in high glucose-induced MPC5 cell and DN mice. Potentially, exosomes secreted from ADSCs can be served as an excellent candidate for DN treatment.

\section{Additional file}

\section{Additional file 1: Figure S1. Isolation of ADSCS. (A-B)Purity}

identification of ADSCs by IF staining and flow cytometryusing CD29,

CD34, CD44, CD45 and CD90 antibodies. Figure S2. Evaluation of cell proliferation and differentiation abilities of ADSCs. (A) Cell viability in passages 3, 6 and 9 of ADSCs were determined by flow cytometry. (B) Cell rate of GO/G1, $S$ and G2/M phase in passages 3, 6 and 9 of ADSCs. (C) Cell viability in passages 3, 6 and 9 of ADSCs were determined by CCK8. (D) APL activity before and after osteogenic medium treatment. (E) Calcium deposit detection by PAS staining before and after osteogenic medium treatment. (F) Adipogenic assessments by oil red $\mathrm{O}$ staining after adipogenic induction medium treatment. Scale bar, $20 \mu \mathrm{m} .{ }^{* *}, p<$ 0.01 . Figure S3. Authentication of the establishing of DN mice. (A-C) Measurement of urine protein, $\mathrm{Scr}$ and BUN in control and spontaneous diabetes mice. (D) PAS staining of renal tissue section in three group mice pretreatment with ADSCs-Exo. (E) Flow chart of exosome injection in control and spontaneous diabetes mice. At 13-weeks old, control and spontaneous diabetes mice were injected with PBS or ADSCs-Exo for additional 12 weeks. ${ }^{* *}, p<0.01$. Figure S4. Authentication of transfection efficiency of miR-486 inhibitor in ADSCs. (A) miR-486 expression was detected by $\mathrm{PPCR}$ after transfection for $24 \mathrm{~h}$ in control ADSC, ADSCmiR486 inhibitor NC and ADSC-miR486 inhibitor. (B) ADSCs-Exo isolated from the above three group ADSCs were identified by WB using CD9, CD63 and CD81 antibodies. (C) miR-486 expression was detected by
qPCR in ADSCs-Exo isolated from the above three group ADSCs. ${ }^{*}, p<$ 0.01 . Figure S5. Authentication of transfection efficiency of smad1 in MPC5 cells. Protein (A) and transcriptional level (B) of smad1 in MPC5 was identified through WB and qPCR methods after transfection for $24 \mathrm{~h} .{ }^{* *} \mathrm{p}$ $<0.01$. (PPTX $3080 \mathrm{~kb}$ )

\section{Abbreviations}

ADSC-Exo: ADSCs-derived exosome; ADSCs: Adipose-derived stem cells; ALP: Alkaline phosphatase; AMPK: AMP-activated protein kinase; BUN: Blood urea nitrogen; DFU: Diabetic foot ulcers; DN: Diabetic nephropathy;

EVs: Extracellular vesicles; GBM: Glomerular basement membrane; IBMX: 3 Isobutyl 1 methylxanthine; MSCs: Mesenchymal stem cells;

mTOR: Mammalian target of rapamycin; Nrf2: Nuclear factor-E2-related factor 2; NTA: Nanosight tracking analysis; PAS: Periodic acid-Schiff; Scr: Serum creatinine; Smad1: Mothers against decapentaplegic homolog 1;

TEM: Transmission electron microscopy; USCs: Urine-derived stem cells

\section{Acknowledgements}

Not applicable.

\section{Funding}

This work was supported by grants from the Major Projects of Science and Technology Department of Zhejiang Province (Grant Number: 2014C030472), the Natural Science Foundation of Zhejiang Province (Grant Number: LY16H050005, LZ17H050001, LY17H050008 and Y18H050024), and the Project of Scientific Research Foundation of Chinese Medicine (Grant Number: 2017ZA010), the General Project of the Medical and Health of Zhejiang Province (Grant Number: 2016 KYB007 and 2017KY227).

Availability of data and materials

Not applicable.

\section{Authors' contributions}

$\mathrm{HQ}$ and $\mathrm{HH}$ conceived the idea. JJ drafted the manuscript. JJ designed and performed the experiments. YF analyzed the data and designed the figures. JG contributed to Western Blotting and IF assay. LZ performed the apoptosis and histological staining assay. YW and $\mathrm{HH}$ provided the clinical breast cancer specimens. All authors discussed the results and edited this manuscript. All authors read and approved the final manuscript.

Ethics approval and consent to participate

The animal care procedures were reviewed and approved by an Institutional Animal Care and Use Committee.

Consent for publication

Not applicable.

\section{Competing interests}

The authors declare that they have no competing interests.

\section{Publisher's Note}

Springer Nature remains neutral with regard to jurisdictional claims in published maps and institutional affiliations.

\section{Author details}

${ }^{1}$ Bone Marrow Transplantation Center, The First Affiliated Hospital, Zhejiang University School of Medicine, Hangzhou, Zhejiang 310003, People's Republic of China. ${ }^{2}$ Department of Nephrology, Zhejiang Provincial People's Hospital, Hangzhou, Zhejiang 310014, People's Republic of China. ${ }^{3}$ People's Hospital of Hangzhou Medical College, Hangzhou, Zhejiang 310014, People's Republic of China. ${ }^{4}$ Department of hematology, The First Affiliated Hospital, Wenzhou Medical University, Fuxue Road 2th, Wenzhou 325000, People's Republic of China. 
Received: 20 November 2018 Revised: 10 February 2019 Accepted: 12 February 2019 Published online: 15 March 2019

\section{References}

1. Piccoli GB, Grassi G, Cabiddu G, Nazha M, Roggero S, Capizzi I, et al. Diabetic kidney disease: a syndrome rather than a single disease. Rev Diabet Stud. 2015;12:87-109.

2. Zhang L, Li R, Shi W, Liang X, Liu S, Ye Z, et al. NFAT2 inhibitor ameliorates diabetic nephropathy and podocyte injury in $\mathrm{db} / \mathrm{db}$ mice. Br J Pharmacol. 2013b;170:426-39.

3. Meyer TW, Bennett PH, Nelson RG. Podocyte number predicts long-term urinary albumin excretion in Pima Indians with type II diabetes and microalbuminuria. Diabetologia. 1999:42:1341-4.

4. Pagtalunan ME, Miller PL, Jumping-Eagle S, Nelson RG, Myers BD, Rennke $H G$, et al. Podocyte loss and progressive glomerular injury in type II diabetes. J Clin Invest. 1997;99:342-8.

5. Fang $L$, Zhou $Y$, Cao H, Wen $P$, Jiang $L$, He W, et al. Autophagy attenuates diabetic glomerular damage through protection of hyperglycemia-induced podocyte injury. PLoS One. 2013;8:e60546

6. Li GB, Li CX, Xia M, Ritter JK, Gehr TWB, Boini K, et al. Enhanced epithelial-tomesenchymal transition associated with lysosome dysfunction in podocytes: role of p62/Sequestosome 1 as a signaling hub. Cell Physiol Biochem. 2015; 35:1773-86

7. Mizushima N, Levine B, Cuervo AM, Klionsky DJ. Autophagy fights disease through cellular self-digestion. Nature. 2008;451:1069-75.

8. Ding DF, You N, Wu XM, Xu JR, Hu AP, Ye XL, et al. Resveratrol attenuates renal hypertrophy in early-stage diabetes by activating AMPK. Am J Nephrol. 2010;31:363-74.

9. Yang Y, Wang J, Qin L, Shou Z, Zhao J, Wang H, et al. Rapamycin prevents early steps of the development of diabetic nephropathy in rats. Am J Nephrol. 2007;27:495-502.

10. Godel M, Hartleben B, Herbach N, Liu S, Zschiedrich S, Lu S, et al. Role of mTOR in podocyte function and diabetic nephropathy in humans and mice. J Clin Invest. 2011;121:2197-209.

11. Wu L, Feng Z, Cui S, Hou K, Tang L, Zhou J, et al. Rapamycin upregulates autophagy by inhibiting the mTOR-ULK1 pathway, resulting in reduced podocyte injury. PLoS One. 2013;8:e63799.

12. Paulini J, Higuti E, Bastos RM, Gomes SA, Rangel EB. Mesenchymal stem cells as therapeutic candidates for halting the progression of diabetic nephropathy. Stem Cells Int. 2016;2016:9521629.

13. Zhang L, Li K, Liu X, Li D, Luo C, Fu B, et al. Repeated systemic administration of human adipose-derived stem cells attenuates overt diabetic nephropathy in rats. Stem Cells Dev. 2013a;22:3074-86.

14. Ni W, Fang Y, Xie L, Liu X, Shan W, Zeng R, et al. Adipose-derived mesenchymal stem cells transplantation alleviates renal injury in streptozotocin-induced diabetic nephropathy. J Histochem Cytochem. 2015; 63:842-53.

15. Massee M, Chinn K, Lim JJ, Godwin L, Young CS, Koob TJ. Type I and II diabetic adipose-derived stem cells respond in vitro to dehydrated human amnion/chorion membrane allograft treatment by increasing proliferation, migration, and altering cytokine secretion. Adv Wound Care (New Rochelle). 2016;5:43-54.

16. Yuan Y, Shi M, Li L, Liu J, Chen B, Chen Y, et al. Mesenchymal stem cellconditioned media ameliorate diabetic endothelial dysfunction by improving mitochondrial bioenergetics via the Sirt1/AMPK/PGC-1alpha pathway. Clin Sci (Lond). 2016;130:2181-98.

17. Hu L, Wang J, Zhou X, Xiong Z, Zhao J, Yu R, et al. Exosomes derived from human adipose mensenchymal stem cells accelerates cutaneous wound healing via optimizing the characteristics of fibroblasts. Sci Rep. 2016;6:32993.

18. Jiang Z-Z, Liu Y-M, Niu X, Yin J-Y, Hu B, Guo S-C, et al. Exosomes secreted by human urine-derived stem cells could prevent kidney complications from type I diabetes in rats. Stem Cell Res Ther. 2016;7:1-13.

19. Johnsen KB, Gudbergsson JM, Skov MN, Christiansen G, Gurevich L, Moos T, et al. Evaluation of electroporation-induced adverse effects on adiposederived stem cell exosomes. Cytotechnology. 2016;68:2125-38.

20. Kamerkar S, LeBleu VS, Sugimoto H, Yang SJ, Ruivo CF, Melo SA, et al. Exosomes facilitate therapeutic targeting of oncogenic KRAS in pancreatic cancer. Nature. 2017:546:498.

21. Chen S, Kang Y, Sun Y, Zhong Y, Li Y, Deng L, et al. Deletion of Gab2 in mice protects against hepatic steatosis and steatohepatitis: a novel therapeutic target for fatty liver disease. J Mol Cell Biol. 2016;8:492-504.
22. Yi M, Zhang L, Liu Y, Livingston MJ, Chen JK, Nahman NS Jr, et al. Autophagy is activated to protect against podocyte injury in adriamycininduced nephropathy. Am J Physiol Renal Physiol. 2017;313:F74-84.

23. Wang Z, Shen Z, Li Z, Duan J, Fu S, Liu Z, et al. Activation of the BMP-BMPR pathway conferred resistance to EGFR-TKls in lung squamous cell carcinoma patients with EGFR mutations. Proc Natl Acad Sci U S A. 2015;112:9990-5.

24. Zhou J, Lee PL, Lee Cl, Wei SY, Lim SH, Lin TE, et al. BMP receptor-integrin interaction mediates responses of vascular endothelial Smad1/5 and proliferation to disturbed flow. J Thromb Haemost. 2013:11:741-55.

25. Lindblom R, Higgins G, Coughlan M, de Haan JB. Targeting mitochondria and reactive oxygen species-driven pathogenesis in diabetic nephropathy. Rev Diabet Stud. 2015;12:134-56

26. Shaw JE, Sicree RA, Zimmet PZ. Global estimates of the prevalence of diabetes for 2010 and 2030. Diabetes Res Clin Pract. 2010;87:4-14.

27. Bruno S, Grange C, Deregibus MC, Calogero RA, Saviozzi S, Collino F, et al. Mesenchymal stem cell-derived microvesicles protect against acute tubular injury. J Am Soc Nephrol. 2009;20:1053-67.

28. Romagnani P. Kidney regeneration: any prospects? Contrib Nephrol. 2011; 170:228-36

29. Bailey AM, Kapur S, Katz AJ. Characterization of adipose-derived stem cells: an update. Curr Stem Cell Res Ther. 2010;5:95-102.

30. Salgado AJ, Reis RL, Sousa NJ, Gimble JM. Adipose tissue derived stem cells secretome: soluble factors and their roles in regenerative medicine. Cur Stem Cell Res Ther. 2010;5:103-10.

31. Li X, Xie X, Lian W, Shi R, Han S, Zhang H, et al. Exosomes from adiposederived stem cells overexpressing Nrf2 accelerate cutaneous wound healing by promoting vascularization in a diabetic foot ulcer rat model. Exp Mol Med. 2018:50:29.

32. Wolf G, Chen S, Ziyadeh FN. From the periphery of the glomerular capillary wall toward the center of disease: podocyte injury comes of age in diabetic nephropathy. Diabetes. 2005;54:1626-34.

33. Daniel J, Klionsky SDE. Autophagy as a regulated pathway of cellular degradation. Science. 2000:290:1717-21.

34. Shintani T, Klionsky DJ. Autophagy in health and disease: a double-edged sword. Science. 2004:306:990-5.

35. Chen JK, Chen J, Neilson EG, Harris RC. Role of mammalian target of rapamycin signaling in compensatory renal hypertrophy. J Am Soc Nephrol. 2005;16:1384-91.

36. Komatsu M, Waguri S, Koike M, Sou YS, Ueno T, Hara T, et al. Homeostatic levels of p62 control cytoplasmic inclusion body formation in autophagydeficient mice. Cell. 2007:131:1149-63.

37. EL Andaloussi S, Mager I, Breakefield XO, Wood MJ. Extracellular vesicles: biology and emerging therapeutic opportunities. Nat Rev Drug Discov, 2013:12:347-57.

38. Katakowski M, Buller B, Zheng X, Lu Y, Rogers T, Osobamiro O, et al. Exosomes from marrow stromal cells expressing miR-146b inhibit glioma growth. Cancer Lett. 2013;335:201-4.

39. Lee HK, Finniss S, Cazacu S, Bucris E, Ziv-Av A, Xiang C, Bobbitt K, Rempel SA, Hasselbach L, Mikkelsen T, Slavin S, Brodie C. Mesenchymal stem cells deliver synthetic microRNA mimics to glioma cells and glioma stem cells and inhibit their cell migration and self-renewal. Oncotarget. 2013;4:346-61.

40. Chen X, Ba Y, Ma L, Cai X, Yin Y, Wang K, et al. Characterization of microRNAs in serum: a novel class of biomarkers for diagnosis of cancer and other diseases. Cell Res. 2008;18:997-1006.

41. Mitchell PS, Parkin RK, Kroh EM, Fritz BR, Wyman SK, Pogosova-Agadjanyan EL, et al. Circulating microRNAs as stable blood-based markers for cancer detection. Proc Natl Acad Sci U S A. 2008:105:10513-8.

42. Valadi H, Ekstrom K, Bossios A, Sjostrand M, Lee JJ, Lotvall JO. Exosomemediated transfer of mRNAs and microRNAs is a novel mechanism of genetic exchange between cells. Nat Cell Biol. 2007;9:654-9.

43. Ghai $\mathrm{V}, \mathrm{Wu} X$, Bheda-Malge A, Argyropoulos CP, Bernardo JF, Orchard T, et al. Genome-wide profiling of urinary extracellular vesicle microRNAs associated with diabetic nephropathy in type 1 diabetes. Kidney Int Rep. 2018.3.555-72 